\title{
Selective recognition of methotrexate by molecularly imprinted polymers
}

Manuela Curcio, Ortensia Ilaria Parisi, Giuseppe Cirillo, Umile Gianfranco Spizzirri, Francesco Puoci, ${ }^{*}$ Francesca lemma, Nevio Picci

"Dipartimento di Scienze Farmaceutiche, Università della Calabria, Edificio Polifunzionale, 87036, Arcavacata di Rende (CS), Italy; tel: 00390984 493151; fax: 00390984 493298; email: francesco.puoci@unical.it

(Received: 17 November, 2008; published: 21 June, 2009)

\begin{abstract}
The aim of this study is the preparation of molecularly imprinted polymers with high selectivity for metotrexate (MTX), a useful antineoplastic drug widely employed in clinical trials. Methacrylic acid, 2-(dimethylamino)ethyl methacrylate and a mixture of them were tested as functional monomers in order to optimize the performance of imprinted materials. The specificity and the selectivity of imprinted polymers was tested by performing rebinding experiments in water at $\mathrm{pH}$ 7.4. The best results were obtained by employing both functional monomers in the polymeric feed. In this way, each monomer interacts with complementary functionalities on template molecule, stabilizing the prepolymerization complex and minimizing the repulsive effects (due to the ionization of functional groups) in the rebinding media.
\end{abstract}

\section{Introduction}

Molecular imprinting is a very useful technique to incorporate specific substrate recognition sites into polymers (Anderson et al. [1], Puoci et al. [2], Yoshimatsu et al. [3]). Molecular recognition characteristics of these polymers are attributed to complementary size, shape, and binding sites imparted to the polymers by the template molecules (Puoci et al. [4], Wang et al. [5]). MIPs are synthetic materials with high recognition properties for a target molecule named template (Mosbach [6]). These specific binding properties must be attributed to specific interactions between the template and the functional groups in the polymeric network (Puoci et al. [7]). The technique to produce MIPs involves arranging the functional monomers around the template to form a prepolymerization complex by both covalent and non-covalent interactions (Cobb et al. [8]). This complex is subsequently radically copolymerized in a solution containing a high ratio of a suitable crosslinker. After copolymerization, the template is removed from the polymer, leaving its imprint in the polymeric structure (Puoci et al. [9]). Usually, different kinds of template for the synthesis of MIPs were used, and our interest was focused on a antineoplastic drug widely used in clinical practice: $\quad$ (S)-2-(4-((2,4-diaminopteridin-6-yl)methyl)(methyl)amino)benzamido) pentanedioic acid, commonly named methotrexate (MTX).

Methotrexate is an antimetabolite that interferes with DNA replication and cell division by inhibiting the enzyme dihydrofolate reductase; it is commonly used in the treatment of various cancers, rheumatoid arthritis, and psoriasis (Gibson et al. [10], Vemulapalli et al. [11]). However, since the cytotoxic effect of MTX is not selective for the cancer cells, it also affects the normal tissues that have a high rate of 
proliferation, including the hematopoietic cells of the bone marrow and the actively dividing cells of the gut mucosa (Zhang et al. [12]). Thus, the efficiency of this agent is often limited by severe side effects and toxic effects. For these reasons, in literature many studies reports on the development of sensitive methods for the monitoring of the concentration of MTX in human body (Frenkel [13], AlvarezFigueroa et Al. [4]).

Chen et al. [15] employed molecularly imprinted solid-phase extraction technique (MISPE) combined with electrochemical oxidation fluorimetry for the determination of methotrexate (MTX) in human serum and urine samples. However, because of the poor stability and solubility of MTX in aprotic and low polarity organic solvent, an analogue trimethoprim was employed as "dummy template" instead of MTX.

In this work, molecularly imprinted polymers were synthesized following the noncovalent approach by using MTX as template.

In the design of an imprinting protocol, particular attention was done in the choice of functional monomers, whose chemical groups, in order to obtain materials with good recognition properties, have to be complementary with those of the template. Functional monomers and template, indeed, are involved in formation of the prepolymerization complex, and the stability of this one is essential for the constitution of imprinted cavities (Puoci et al. [16]).

In our study, considering the functionalities of the template molecule, methacrylic acid (MAA), 2-(dimethylamino)ethyl methacrylate (MADAME) and a mixture of MAA and MADAME in different molar ratios were employed. The selectivity properties of obtained materials were evaluated by using folic acid (FA), a MTX analogue molecule (Figure 1).<smiles>[R14][X]c1nc(N)nc2ncc(CN(C)c3ccc(C(=O)N[Y]([H])([H])C(CCC(=O)O)C(=O)O)cc3)nc12</smiles><smiles>[H][Y]c1nc(O)c2nc(CNc3ccc(C(=O)NC(CCC(=O)O)C(=O)O)cc3)cnc2n1</smiles>

Fig. 1. Chemical structure of methotrexate (MTX) and folic acid (FA). 


\section{Results and discussion}

As explained before, the choice of the functional monomer(s) plays a key role in the preparation of an effective molecularly imprinted polymer. Because the non-covalent imprinting approach was employed, the interactions between functional monomers and template are based on $\mathrm{H}$-bonds or ionic interactions (Beltran at al. [17]). In our

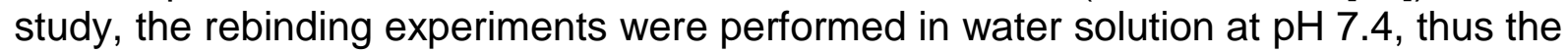
ionization behaviour of both template and polymer structures strongly influences the performance of the imprinted polymers.

MTX is the prototype folate antagonist cytotoxic drug. Its molecule is made up of a heterocyclic portion (a 2,4-diaminosubstituted pterine ring) linked to a aminobenzoil portion, which is, in turn, an amide bonded to a glutamic acid unit (Hall et al. [18]). Hence, MTX is a polyelectrolyte carrying two carboxyl groups, with dissociation constants (pKa) of 3.36 ( $\alpha$-carboxyl) and 4.70 ( $\mathrm{y}$-carboxyl), and a number of potentially protonated nitrogen functions, the most basic of which, presumably, the guanidinic N-1 on the pterine ring (pKa 5.71) (de Faria et al. [19]).

It follows that, as shown in Figure 2, at $\mathrm{pH}$ of the rebinding media (7.4), the employed functional monomers establishes interactions with different parts of the template molecule. These interactions are due in part to hydrogen and partly to ionic bonds. Specifically, acidic monomer (MAA) bound to pteridine moiety, while basic monomer (MADAME) interacts with the carboxyl groups of glutamic acid.

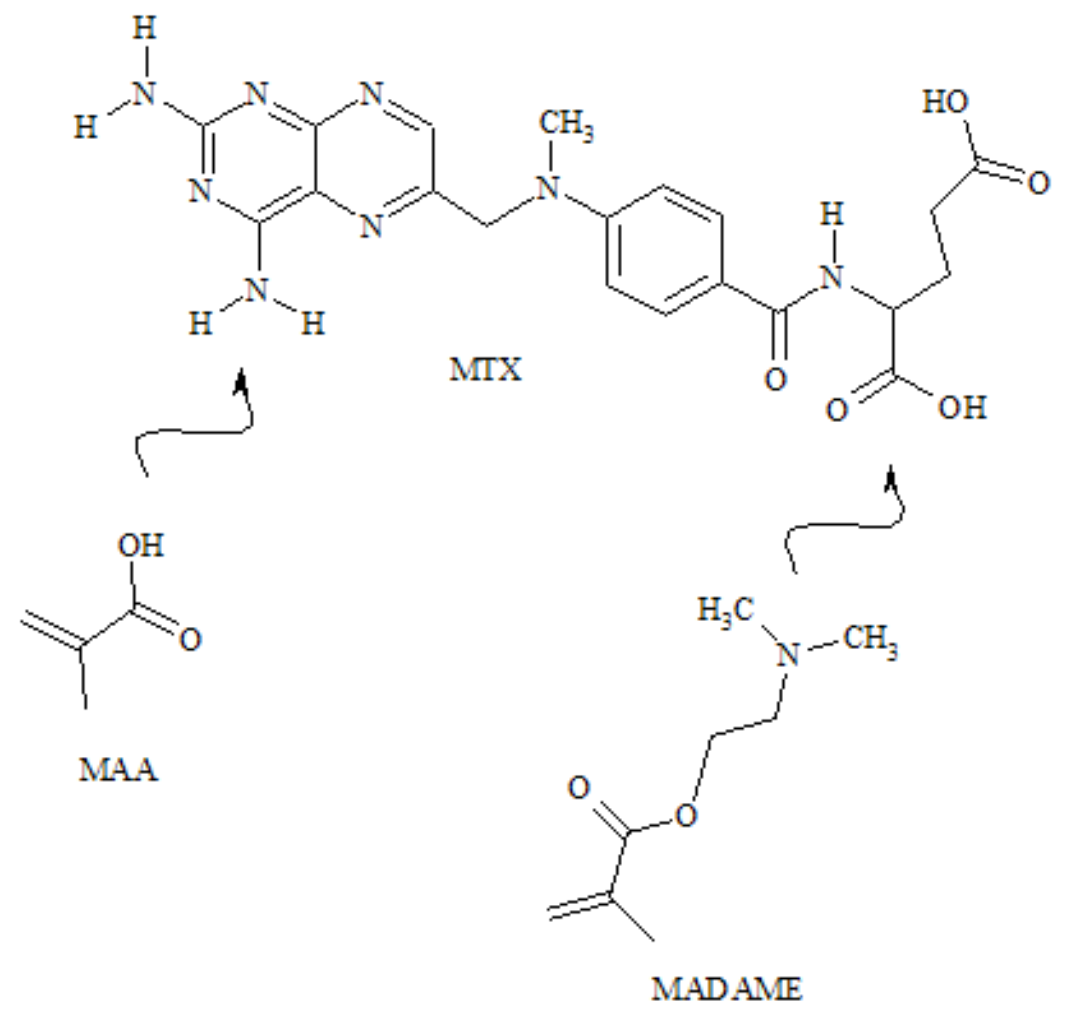

Fig. 2. Interaction model between template and functional monomers.

The imprinting effect of synthesized materials was evaluated by binding experiments in which amounts of polymeric particles were incubated with $0.05 \mathrm{mM} \mathrm{MTX}$ solutions for 24 hours. These experiments were performed in buffered water solution at $\mathrm{pH}=$ 7.4 . 
In Table 1 are reported MTX bound (\%) and the relative $\alpha$ values.

Tab. 1. Bound (\%) MTX and FA by imprinted and non-imprinted polymers.

\begin{tabular}{lllll}
\hline Polymers & $\begin{array}{l}\text { \% bound } \\
\text { MTX }\end{array}$ & $\begin{array}{l}\text { \% bound } \\
\text { FA }\end{array}$ & $\boldsymbol{\alpha}_{\text {MTX }}$ & $\boldsymbol{\alpha}_{\text {FA }}$ \\
\hline MIP1 & $37 \pm 1.2$ & $7 \pm 0.5$ & \multirow{2}{*}{1.19} & \multirow{2}{*}{1.17} \\
NIP1 & $31 \pm 1.3$ & $6 \pm 0.6$ & & \\
\hline MIP2 & $14 \pm 1.1$ & $10 \pm 0.7$ & \multirow{2}{*}{0.93} & 0.91 \\
NIP2 & $15 \pm 0.9$ & $11 \pm 0.9$ & & \\
\hline MIP3 & $26 \pm 1.1$ & $36 \pm 0.9$ & \multirow{2}{*}{1.13} & \multirow{2}{*}{1.16} \\
NIP3 & $23 \pm 0.8$ & $31 \pm 0.7$ & & \\
\hline MIP4 & $64 \pm 0.9$ & $67 \pm 1.0$ & \multirow{2}{*}{1.08} & \multirow{2}{*}{1.06} \\
NIP4 & $59 \pm 0.7$ & $63 \pm 1.3$ & & \\
\hline MIP5 & $54 \pm 0.7$ & $31 \pm 0.7$ & \multirow{2}{*}{1.13} & \multirow{2}{*}{1.15} \\
NIP5 & $48 \pm 1.1$ & $27 \pm 0.8$ & & \multirow{2}{*}{1.51} \\
\hline MIP6 & $80 \pm 1.0$ & $59 \pm 1.3$ & & \multirow{2}{*}{20} \\
NIP6 & $53 \pm 1.3$ & $49 \pm 1.1$ & & \\
\hline
\end{tabular}

Polymers in which only MAA was employed as functional monomer (MIP1 and MIP2) did not show any significant imprinting effect. In addition, by enhancing the amount of functional monomer (MIP2) in the polymeric feed, percentages of bound template significantly decreased. This behaviour is ascribable to the increasing of repulsive forces between ionized carboxylic groups of MAA and MTX in the rebinding media $(\mathrm{pH} 7.4)$ which overlay the attractive forces between functional monomer and pterine moiety of MTX.

In order to avoid these negative interactions between carboxylic groups, MIP3 and MIP4 were synthesized by using a functional monomer presenting chemical groups that are able to coordinate the glutammic acid moiety of MTX. For this purpose, a basic monomer such as MADAME was employed. As shown, also in this case the obtained resins present no significant imprinting effect. It was observed that, by increasing the amount of functional monomer, a considerable increase of the non specific interactions of template-polymeric matrices was found.

Considering the results obtained by using single monomers, MIP5 and MIP6 were synthesized by employing both acidic and basic monomers in the polymeric feed and using two different stoichiometric ratios MADAME/MAA. In this way, each monomer interacts with complementary functionalities on template molecule (Figure 2), stabilizing the prepolymerization complex and minimizing the repulsive effects (due to the ionization of functional groups) in the rebinding media.

The best result was obtained by using the highest ratio MADAME/MAA (MIP6). As shown in Table 1, indeed, the imprinting effect was improved, as highlighted by increase of MTX bound (\%) observed in moving from MIP5 to MIP6, and by unchanged value of aspecific bonds.

In literature, different approaches were applied to make a quantitative determination of the imprinting effect (Ye et al. [20]). The imprinting efficiency $(\alpha)$ is the easiest way to highlight the recognition properties in a MIP. In our work, $\alpha_{\text {MTX }}$ was determined as the ratio between the amount (\%) of MTX bound by MIP and NIP, respectively (Gore 
et al. [21]). By comparing $\alpha_{M T X}$ values (Table 1), it is clear that MIP6 is the most effective polymer in selective recognition of MTX in a simulating physiological fluid.

To evaluate cross-reactivity of imprinted polymers towards MTX analogue molecule, the same binding experiments were performed using FA, which differs from MTX in a substituent on pterine moiety $(-\mathrm{OH}$ instead of $-\mathrm{NH} 2)$ and in the amino group of the $\mathrm{p}$ aminobenzoil portion (Figure 1).

The most effective imprinted polymers in recognition of the template (MIP6), is characterized by a lower affinity towards the analogue FA. The binding percentages of MIP6 decrease $21 \%$ moving from MTX to FA, while they are unchanged in NIP6 case.

As reported for MTX, $\alpha_{F A}$ was also determined as ratio between FA bound by MIP and NIP, respectively. In MIP6, a lower $\alpha_{\mathrm{FA}}$ than $\alpha_{\mathrm{MTX}}$ shows the chemical and spatial complementarities of MIP binding sites towards the template.

\section{Experimental part}

\section{Materials}

Ethylene glycol dimethacrylate (EGDMA), methacrylic acid (MAA), 2(dimethylamino)ethyl methacrylate (MADAME), 2,2'-azoisobutyronitrile (AIBN), methotrexate (MTX), folic acid (FA), sodium hydrogen carbonate, hydrochloric acid $(37 \% \mathrm{w} / \mathrm{w})$, sodium hydrogen phospate, disodium hydrogen phosphate, dimethyl sulfoxide (DMSO) and acetonitrile were obtained from Aldrich. All solvents were reagent grade or HPLC-grade and used without further purification and they were provided by Fluka Chemie.

MAA and MADAME were purified before use by distillation under reduced pressure.

\section{Instrumentation}

The liquid chromatography consisted of an Jasco BIP-I pump and Jasco UVDEC$100-V$ detector set at $290 \mathrm{~nm}$. A $25 \times 0.4 \mathrm{~mm}$ C4 Kromasil 100 column, particle size $5 \mu \mathrm{m}$ (Teknocroma, Barcellona, Spain) was employed. The mobile phase was phosphate buffer $0.01 \mathrm{M}, \mathrm{pH} \mathrm{7,4/acetonitrile}(9 / 1, \mathrm{v} / \mathrm{v})$ run isocratically at a flow rate of $1 \mathrm{~mL} / \mathrm{min}$ and at room temperature.

\section{Synthesis of methotrexate imprinted polymers}

Molecularly Imprinted Polymers (MIPs) were prepared by bulk polymerization using methotrexate as template and according to the non-covalent imprinting approach. Briefly, MTX and functional monomer(s) were dissolved in $4.7 \mathrm{ml}$ of DMSO in a thickwalled glass tube. The tube was sonicated for $6 \mathrm{~min}$ in a sonicating water bath and then EGDMA and AIBN were added. The polymerization mixture was bubbled with nitrogen, sonicated for $10 \mathrm{~min}$ and thermo-polymerized under a nitrogen atmosphere for $24 \mathrm{~h}$ at $60{ }^{\circ} \mathrm{C}$. The resultant bulk rigid polymers were crushed, grounded into powder and sieved through a $63 \mathrm{~nm}$ stainless steel sieve. The sieved MIPs materials were collected and the very fine powder, suspended in the supernatant solution (acetone), was discarded. The obtained MIPs materials were extracted by Soxhlet apparatus with $200 \mathrm{ml}$ of methanol for $48 \mathrm{~h}$ and successively they were dried under vacuum overnight at $40{ }^{\circ} \mathrm{C}$. The extracted imprinted polymers were boiled in $250 \mathrm{ml}$ of a saturated solution of sodium hydrogen carbonate for $2 \mathrm{~h}$ and then neutralized 
using $\mathrm{HCl} 0.1 \mathrm{~N}$ and distilled water. The resultant particles were dried under vacuum overnight at $40^{\circ} \mathrm{C}$. The washed MIPs materials were checked to be free of MTX and any other compound by HPLC analysis. Blank polymers that act as a control were synthesized under the same reaction conditions but in absence of the template. The molecular ratios of the different prepared polymers are shown in Table 2.

Tab. 2. Polymerization feeds composition.

\begin{tabular}{|c|c|c|c|c|c|c|c|}
\hline Polymers & $\begin{array}{c}\text { MTX } \\
(g)\end{array}$ & $\begin{array}{l}\text { MAA } \\
(g)\end{array}$ & $\begin{array}{l}\text { MADAME } \\
\text { (g) }\end{array}$ & $\begin{array}{l}\text { EGDMA } \\
(\mathrm{g})\end{array}$ & $\begin{array}{c}\text { MTX:MAA:MADAME: } \\
\text { EGDMA }\end{array}$ & $\begin{array}{l}\text { DMSO } \\
(\mathrm{ml})\end{array}$ & $\begin{array}{c}\text { AIBN } \\
(g)\end{array}$ \\
\hline $\begin{array}{l}\text { MIP1 } \\
\text { NIP1 }\end{array}$ & 0.14 & 0.43 & - & 3.10 & $1: 16: 0: 50$ & 4.70 & 0.07 \\
\hline $\begin{array}{l}\text { NIP1 } \\
\text { MIP2 }\end{array}$ & $\frac{-}{0.14}$ & & & & & & \\
\hline NIP2 & 0.14 & 0.86 & - & 3.10 & $1: 32: 0: 50$ & 4.70 & 0.07 \\
\hline \begin{tabular}{|l|} 
MIP3 \\
NIP3 \\
\end{tabular} & $\begin{array}{c}0.14 \\
-\end{array}$ & - & 0.79 & 3.10 & $1: 0: 16: 50$ & 4.70 & 0.07 \\
\hline $\begin{array}{l}\text { MIP4 } \\
\text { NIP4 } \\
\end{array}$ & $\begin{array}{c}0.14 \\
-\end{array}$ & - & 1.57 & 3.10 & $1: 0: 32: 50$ & 4.70 & 0.07 \\
\hline $\begin{array}{l}\text { MIP5 } \\
\text { NIP55 }\end{array}$ & $\begin{array}{c}0.14 \\
-\end{array}$ & 0.43 & 0.79 & 3.10 & $1: 16: 16: 50$ & 4.70 & 0.07 \\
\hline $\begin{array}{l}\text { MIP6 } \\
\text { NIP6 } \\
\end{array}$ & $\begin{array}{c}0.14 \\
-\end{array}$ & 0.43 & 1.57 & 3.10 & 1:16:32:50 & 4.70 & 0.07 \\
\hline
\end{tabular}

\section{Binding experiments}

Evaluation of the capacity of the polymeric materials to recognize and bind MTX was performed by rebinding experiments in phosphate buffer solution ( $\mathrm{pH}$ 7.4). Briefly, 50 $\mathrm{mg}$ of polymer particles were mixed with $1 \mathrm{ml}$ MTX solution $(0.05 \mathrm{mM})$ in a $1 \mathrm{ml}$ Eppendorf and sealed. The Eppendorf were oscillated by a wrist action shaker (Burrell Scientific) in a water bath at $37 \pm 0.5{ }^{\circ} \mathrm{C}$ for $24 \mathrm{~h}$. Then the mixture was centrifuged for $10 \mathrm{~min}$ (10000 rpm) in an $\mathrm{ALC}^{\circledR}$ microcentrifugette ${ }^{\circledR} 4214$ and the MTX concentration in the liquid phase was measured by HPLC. The amount of MTX bound to the polymer was obtained by comparing its concentration in the MIPs samples to the NIPs samples. The same experiments were also performed using folic acid solution and they were repeated five times.

\section{Acknowledgements}

This work was financially supported by University funds.

\section{References}

[1] Anderson, R. A.; Ariffin, M. M.; Cormack, P. A. G.; Miller, E. I. Forensic Sci. Int. 2008, 174, 40.

[2] Puoci, F.; lemma, F.; Picci, N. Current Drug Deliv. 2008, 5, 85.

[3] Yoshimatsu, K.; Reimhult, K.; Krozer, A.; Mosbach, K.; Sode, K.; Ye, L. Anal. Chim. Acta 2007, 584, 112.

[4] Puoci, F.; Curcio, M.; Cirillo, G.; Iemma, F.; Spizzirri, U. G.; Picci, N. Food Chem. 2008, 106, 836.

[5] Wang, J.; Cormack, P. A. G.; Sherrington, D. C.; Khoshdl, E. Pure Appl. Chem. 2007, 79, 1505. 
[6] Mosbach, K.; Sci. Am. 2006, 295, 86

[7] Puoci, F.; lemma, F.; Cirillo, G.; Picci, N.; Matricardi, P.; Alhaique, F. Molecules 2007, 12, 805.

[8] Cobb, Z.; Sellergren, B.; Andersson, L. I. Analyst 2007, 132, 1262.

[9] Puoci, F.; Cirillo, G.; Curcio, M.; Iemma, F.; Parisi, O. I.; Castiglione, M.; Picci, N. Drug Deliv. 2008, 15, 253.

[10] Gibson, R. J.; Bowen, J. M.; Keefe, D. M. K. Cancer Treat. Rev. 2008, 34, 476.

[11] Vemulapalli, V.; Banga, A. K.; Friden, P. M. Drug Deliv. 2008, 15, 437.

[12] Zhang, L.; Russell, D.; Conway, B. R.; Batchelor, H. Crit. Rev. Ther. Drug 2008, 25, 259.

[13] Frenkel, V. Adv. Drug Deliver. Rev. 2008, 60, 1193.

[14] Alvarez-Figueroa, M. J.; Blanco-Mendez, J. Int. J. Pharm. 2001, 57, 215.

[15] Chen, S.; Zhang, Z. Spectrochim. Acta A 2008, 36, 70.

[16] Puoci, F.; Cirillo, G.; Curcio, M.; lemma, F.; Spizzirri, U. G.; Picci, N. Anal. Chim. Acta 2007, 593, 164.

[17] Beltran, A.; Caro, E.; Marcè, R. M.; Cormack, P. A. G.; Sherrington, D. C.; Borrul, F. Anal. Chim. Acta 2007, 597, 6.

[18] Hall, A. J.; Quaglia, M.; Manesiotis, P.; De Lorenzi, E.; Sellergren, B. Anal. Chem. 2006, 78, 8362.

[19] de Faria, T. J.; de Campos, A. M.; Lemos Senna, E. Macromol. Symp. 2005, 229, 228.

[20] Ye, L.; Mosbach, K. Chem. Mater. 2008, 20, 859.

[21] Gore, M. A.; Karmalkar, R. N.; Kulkarni, M. G. J. Chromatogr. B 2004, 804, 211. 